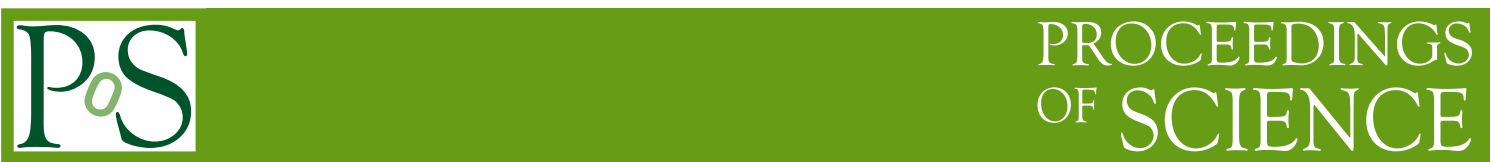

\title{
Measurements of the Higgs properties at ATLAS
}

\author{
Patricia Conde Muíño*t \\ CERN, Switzerland \\ E-mail: Patricia.Conde.Muino@cern.ch
}

\begin{abstract}
The present constraints from electroweak radiative corrections suggest that the low mass region will be particularly interesting in future Higgs boson searches. In this paper, the discovery potential of a search of a Standard Model Higgs boson in the mass range between 110 and $190 \mathrm{GeV}$ by the ATLAS experiment at the LHC is presented, as a function of collected luminosity.

As soon as a significant signal in one of the Higgs decay modes is observed, it will be necessary to do a precise measurement of its parameters. Such measurements should give further insigths into the electroweak symmetry breaking mechanism and into the way the Higgs boson(s) couples to fermions and bosons. The precision that can be achieved at the LHC in the determination of important Higgs boson parameters is also presented in this paper. The measurements discussed are the mass, the width, the spin and the couplings to bosons and fermions. The later measurements use all the possible channels in which the Higgs boson can be observed, including the production via the vector boson fusion process.
\end{abstract}

International Europhysics Conference on High Energy Physics

July 21st - 27th 2005

Lisboa, Portugal

\footnotetext{
* Speaker.

On behalf of the ATLAS Collaboration
} 

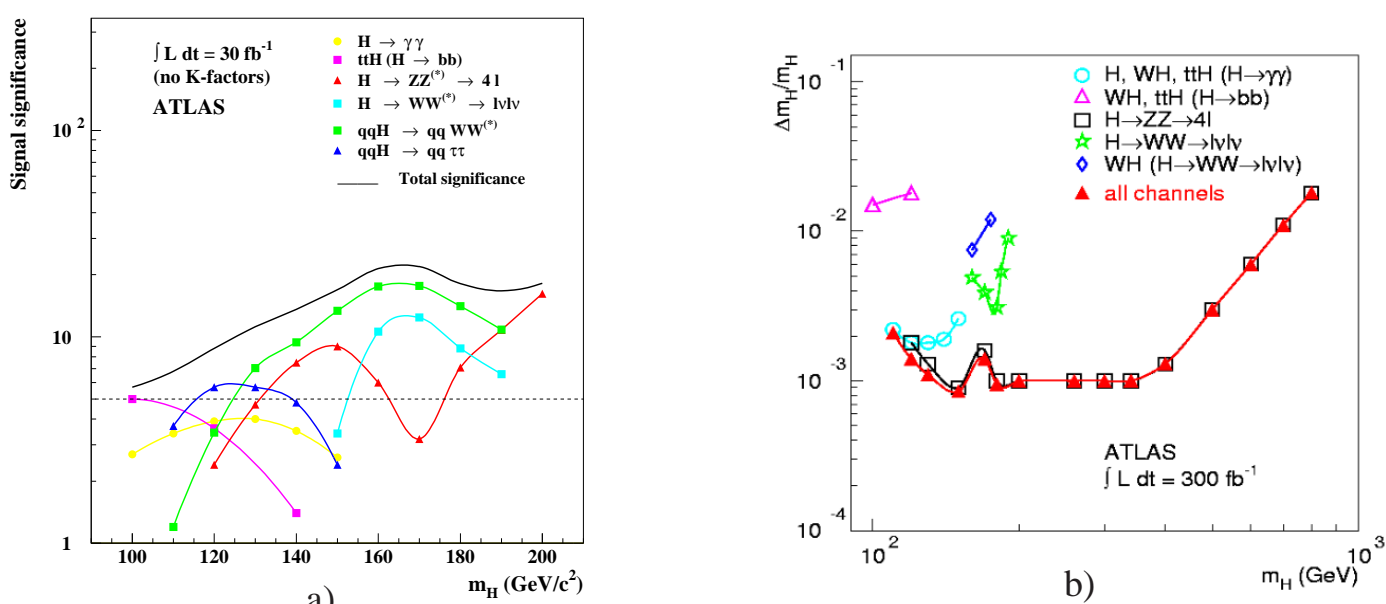

Figure 1: a) Potential of the ATLAS experiment to discover a SM Higgs boson as a function of the Higgs mass. The vector boson fusion processes are indicated as qqH. b) Accuracy on the Higgs mass measurement as a function of the Higgs mass.

\section{Atlas potential to discover the Higgs boson}

The main production mechanism for a SM Higgs is the gluon-gluon fusion, for all the possible Higgs mass range. The second dominant production channel is the vector boson fusion (VBF), produced by the radiation of the vector bosons from the initial state quarks. Although for masses $m_{H}<2 m_{Z}$ this channel contributes to $20 \%$ of the total Higgs production cross section, it provides very clear signatures that can be used to discriminate the signal: two forward jets coming from the initial state quarks and the lack of hadronic activity in the central part of the detector, due to the absence of color exchange between the quarks. Other channels, like associated production, also provide clean signatures that might be enough for a discovery, even having very low cross sections.

The potential of the ATLAS experiment to discover a Standard Model Higgs boson was studied for several different decay channels. The signal significance that can be achieved for $30 \mathrm{fb}^{-1}$ of integrated luminosity, in the mass range between $90-200 \mathrm{GeV}$, is displayed in figure 1 a) as a function of the Higgs mass. The VBF channels obtain the best significance for masses between 120 and $180 \mathrm{GeV}$ [1]. Above the $\mathrm{Z}$ pair production threshold, the dominant channel is $H \rightarrow Z Z \rightarrow 4 l$ [2]. This figure demonstrates that ATLAS is able to discover a SM Higgs boson with high significance for any allowed SM Higgs mass, during the first years of data taking.

The precision that can be achieved in the measurement of the Higgs mass is displayed in figure $1 \mathrm{~b})$ as a function of the Higgs mass, for the full ATLAS luminosity $\left(300 \mathrm{fb}^{-1}\right)$. The best accuracy, of about $0.1 \%$ is obtained for the $H \rightarrow Z Z \rightarrow 4 l$ channel for most of the mass range. For low Higgs masses, the $H \rightarrow \gamma \gamma$ also contributes significantly to improve the mass precision. The error in the mass measurement is dominated by the $0.1 \%$ systematic uncertainty in the lepton/photon energy scale. For large Higgs masses $\left(m_{H}>500 \mathrm{GeV}\right)$, the accuracy is limited by the intrinsic Higgs boson width. 

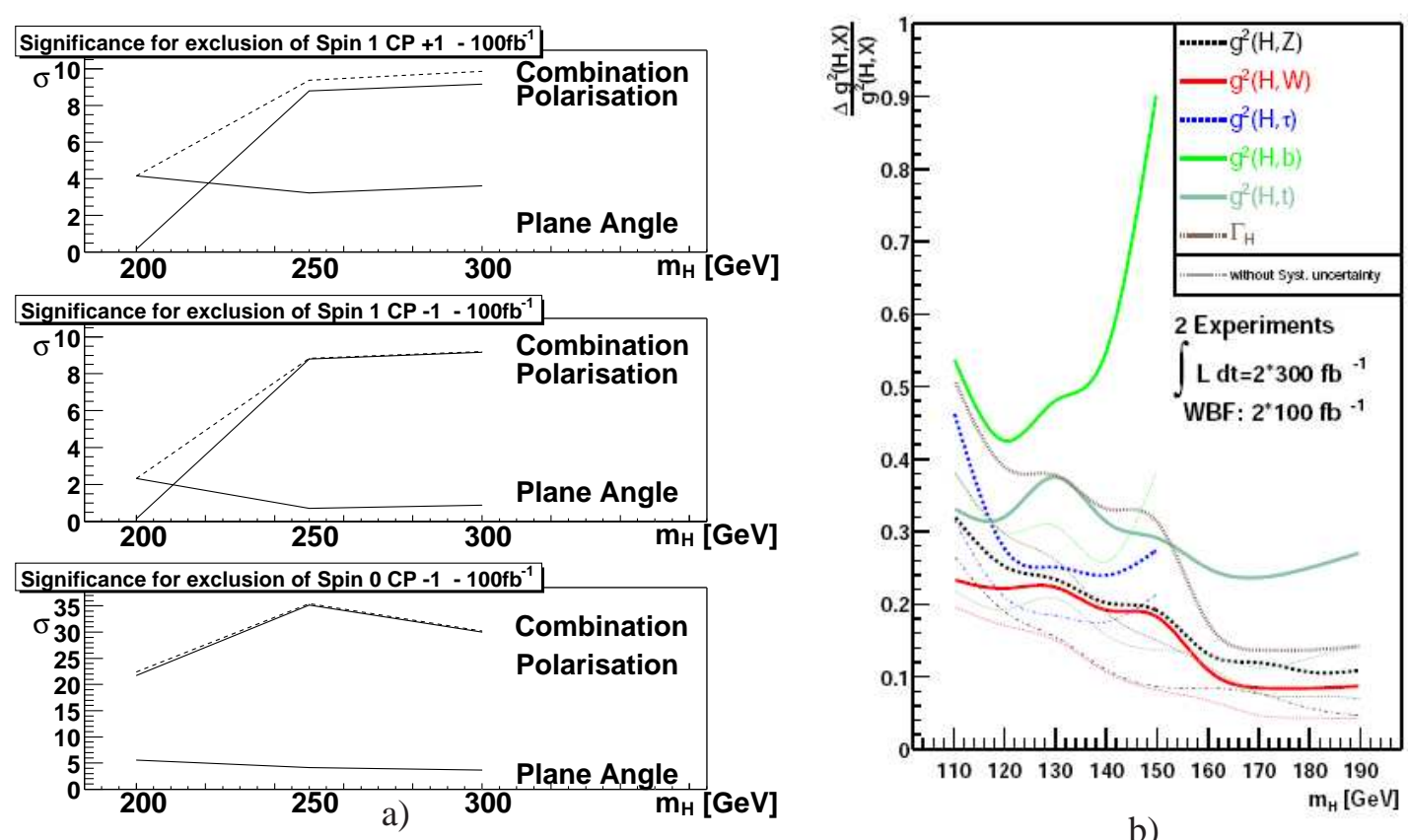

Figure 2: a) Overall significance for the exclusion of the non standard spin and CP-eigenvalue of the Higgs boson, as a function of the Higgs mass. b) Expected relative precision of the Higgs couplings squared as a function of the Higgs boson mass.

\section{Higgs boson spin and $\mathrm{CP}$}

Once a convincing Higgs signal is found, one has to demonstrate whether it is actually a Higgs boson. In order to do that, the properties of this particle have to be measured, and in particular its spin and parity or its couplings to other SM particles (see next section).

The spin and $\mathrm{CP}$ of the Higgs boson can be studied by looking at the angular distributions of the leptons in the decay channel $H \rightarrow Z Z \rightarrow 4 l$ and comparing them with the expectations for a particle with the same mass but different spin and CP quantum numbers. In the study presented here [3], two angular distributions were considered: the cosine of the polar angle of the decay leptons relative to the $\mathrm{Z}$ boson and the angle between the decay planes of the $\mathrm{Z}$ boson in the Higgs rest frame. The final significance that ATLAS can reach with this analysis to exclude non standard spin and CP quantum numbers is displayed in figure 2 a) as a function of the Higgs boson mass, for an integrated luminosity of $100 \mathrm{fb}^{-1}$ and for a mass range between 200 and $300 \mathrm{GeV}$. For large masses, the polarization variable is enough to rule out non standard spin and CP hypothesis already with $100 \mathrm{fb}^{-1}$ of integrated luminosity. For smaller masses, the decay plane angle distribution contributes significantly to exclude the $\operatorname{spin}=1$ hypothesis. To rule out the $\operatorname{spin}=1, \mathrm{CP}=-1$ hypothesis, the full ATLAS luminosity will be necessary.

\section{Higgs boson couplings}

The Higgs couplings determine its production cross sections for the different production mechanisms and the branching ratios of its decays. A maximum likelihood method taking into account 
the measurements of the rates for the different decay channels was developed in order to extract the Higgs coupling constants[4]. Due to the fact that the total width of the Higgs is not observable at ATLAS, only the ratios of the Higgs couplings can be extracted if no additional assumptions are made. With some soft theoretical assumptions, however, also the couplings can be measured. In the results presented here, it was assumed that the strength of the Higgs coupling constants does not exceed the SM values: $\Gamma_{V} \leq \Gamma_{V}^{S M}$, with $\mathrm{V}=\mathrm{W}, \mathrm{Z}$. This assumption is valid for models with any number of Higgs doublets, with or without Higgs singlet, including the Minimal Supersymmetric Standard Model (MSSM).

The likelihood method takes into account all the observable signals, the expected statistical uncertainties in the signal and in the background and the systematic uncertainties in the luminosity, the background estimation and the reconstruction. It allows for undetected Higgs decays and additional contributions to the loop induced Higgs couplings due to non SM particles. The relative precision that can be achieved in the measurements of the different Higgs couplings squared is displayed in figure $2 \mathrm{~b}$ ) as a function of the Higgs mass, for the full LHC luminosity. The dotted lines show the expected relative precision when the systematic uncertainties are not taken into account, demonstrating that any improvement in the systematic uncertainties will lead to an improvement also in the coupling constants measurements. The accuracies range between 20-40\% for Higgs masses below $150 \mathrm{GeV}$. Above the $\mathrm{W}$ pair production threshold, the systematic uncertainties of the Higgs couplings to $\mathrm{W}$ and $\mathrm{Z}$ decreases to $\sim 10 \%$. For a Higgs mass below $140 \mathrm{GeV}$ the main contribution to the systematic error comes from the background normalization of the $H \rightarrow b \bar{b}$ channel that has a small signal to background ratio.

\section{Summary and conclusions}

The ATLAS experiment will be able to detect a Higgs signal with high significance already for $30 \mathrm{fb}^{-1}$ of integrated luminosity, for all the Higgs mass range. The expected accuracy on the Higgs mass is about $0.1 \%$ for $m_{H}<400 \mathrm{GeV}$. After the discovery, precise measurements of its properties have to be done in order to demonstrate that the signal is in fact the Higgs boson. Recent studies have shown that non SM spin and CP hypothesis can be ruled out for $m_{H}>200 \mathrm{GeV}$ with $100 \mathrm{fb}^{-1}$ of luminosity. The squared coupling constants of the Higgs to the bosons and fermions can be measured with a precision that ranges between 10 and 50\%, for the full LHC luminosity.

\section{References}

[1] S. Asai et al., Prospects for the search for a standard model Higgs boson in ATLAS using vector boson fusion, Eur. Phys. J. C 32S2 (2004) 19 [arXiv:hep-ph/0402254].

[2] ATLAS Collaboration, Detector and Physics Performance Technical Design Report, CERN/LHCC/99-14 (1999).

[3] C. P. Buszello, I. Fleck, P. Marquard, J. J. van der Bij,Prospective analysis of Spin and CP-sensitive variables in $H \rightarrow Z Z \rightarrow 4$ l at the LHC, Eur. Phys. J. C 32 (2004) 209.

[4] M. Duhrssen, S. Heinemeyer, H. Logan, D. Rainwater, G. Weiglein and D. Zeppenfeld, Extracting Higgs boson couplings from LHC data, Phys. Rev. D 70 (2004) 113009 [arXiv:hep-ph/0406323]. M. Duhrssen, Prospects for the measurement of Higgs boson coupling parameters in the mass range from 110-190 GeV/c ${ }^{2}$, ATLAS note, ATLAS-PHYS-2003-030. 